\title{
Design of Complex-Valued Variable FIR Digital Filters and Its Application to the Realization of Arbitrary Sampling Rate Conversion for Complex Signals
}

\author{
K. M. Tsui, S. C. Chan, Member, IEEE, and K. W. Tse
}

\begin{abstract}
This brief studies the design of complex-valued variable digital filters (CVDFs) and their applications to the efficient arbitrary sample rate conversion for complex signals. The design of CVDFs using either the minimax or least-squares criteria is formulated as a convex optimization problem and solved using the second-order cone programming (SOCP). In addition, linear and convex quadratic inequality constraints can be readily incorporated. Design examples are given to demonstrate the effectiveness of the proposed approach.
\end{abstract}

Index Terms-Complex-valued variable digital filter (CVDF) design, flatness and peak error constraints, low delay, sampling rate conversion, second-order cone programming (SOCP).

\section{INTRODUCTION}

$\mathbf{V}$ ARIABLE DIGITAL FILTERS (VDFs) [1] are digital filters with controllable spectral characteristics such as variable cutoff frequency, adjustable passband width [2], [3], controllable fractional delay [4]-[7], etc. They are useful in arbitrary sample rate changers [8], digital synchronizers [9], and other applications involving on-line tuning of frequency characteristics. The least-squares (LS) design criterion is commonly used in the design of Farrow-based finite-impulse response (FIR) VDFs [2], [10], [11] because it only involves the solution of a system of linear equation. The linear programming technique [3], [7] has also been proposed to design variable digital filters having linear-phase characteristics and minimax design errors. Another useful method based on semidefinite programming (SDP) was recently proposed in [8], which is able to design both linear-phase and low-delay VDFs in either minimax or least-square (LS) criterion. Most VDFs considered so far have real-valued coefficients. In processing bandpass signals and array signal processing, the signals are usually complex-valued. Therefore, the processing of complex-valued signals using VDFs is of considerable interests.

In this brief, we mainly focus on the design problem of complex-valued VDFs (CVDFs), possibly with prescribed number of zeros and peak error constraints in the stopband. Constraints

Manuscript received June 23, 2004; revised November 15, 2004. This paper was presented in part at the IEEE International Symposium on Circuits and Systems, Bangkok, Thailand, May 2003. This paper was recommended by Associate Editor C.-T. Lin.

The authors are with the Department of Electrical and Electronic Engineering, The University of Hong Kong, Hong Kong (e-mail: kmtsui@eee.hku.hk; scchan@eee.hku.hk; kwtse@eee.hku.hk).

Digital Object Identifier 10.1109/TCSII.2005.850405 like multiple zeros are usually desirable in sample rate converters for the suppression of alias components, and the design of wavelet basis. On the other hand, peak error constraints are useful to limit the sidelobes and undesirable peaks in filters with wide unconstrained transition band. Both LS and minimax design criteria will be considered. These design problems are formulated as a convex programming problem and is solved using second-order cone programming (SOCP) [12]-[14]. Alternatively, another efficient convex optimization tool called SDP [15] can also be used. In SOCP, a linear objective function of the design variable vector $\boldsymbol{x}$ (an $N$ th vector), $\boldsymbol{c}^{T} \boldsymbol{x}$, is minimized subject to a series of quadratic constraints having the form $\boldsymbol{c}_{i}^{T} \boldsymbol{x}-$ $k_{i} \geq\left\|\boldsymbol{Q}_{i} \boldsymbol{x}-\boldsymbol{p}_{i}\right\|_{2}$. That is

$$
\min _{\boldsymbol{x}} \boldsymbol{c}^{T} \boldsymbol{x} \quad \text { subject to } \boldsymbol{c}_{i}^{T} \boldsymbol{x}-k_{i} \geq\left\|\boldsymbol{Q}_{i} \boldsymbol{x}-\boldsymbol{p}_{i}\right\|_{2}
$$

for $i=1, \ldots, N_{c}$, where $\boldsymbol{c}$ and $c_{i}$ are $(N \times 1)$ constant vectors, $\boldsymbol{p}_{i}$ 's are $\left(m_{i} \times 1\right)$ constant vectors, $\boldsymbol{Q}_{i}$ 's are $\left(m_{i} \times N\right)$ constant matrices, $k_{i}$ are constants, and $\|\cdot\|_{2}$ denotes the Euclidean norm. The main advantage of using SOCP and SDP is that the problem is convex and the global optimal solution, if it exists, is guaranteed. Furthermore, a wide variety of constraints such as linear equalities and inequalities constraints and convex quadratic constraints can be imposed readily to meet different design objectives and constraints. Interested readers are referred to [12]-[14] for more details.

In this brief, the constraints of prescribed number of zeros for the CVDFs are formulates as a set of linear equality constraints, which are imposed separately to each subfilter in the Farrowbased implementation of the VDFs [4]. Within the SOCP (or SDP) framework, these linear equality and convex quadratic inequality constraints can be integrated together for the optimal design of CVDFs with minimax and LS errors. Unlike the linear programming approach, the proposed approach is also applicable to the design of CVDF with lower passband group delay than its linear-phase counterpart with the same filter length. Design results show that the proposed method offers an attractive alternative to traditional methods because of its optimality, generality and flexibility.

Another objective of this brief is the application of the proposed CVDFs to the realization of sampling rate converter (SRC), which is an important component in software radio receivers [16]. The basic idea of a VDF-based SRC is to provide variable fractional delay in the passband and additional attenuation in the stopband. Therefore, arbitrary sampling rate 
conversion can be achieved by tuning the control parameter in the Farrow's structure. In this brief, the approach in [8] is extended to complex-valued input signals possibly with an asymmetric magnitude and phase characteristics. It should be noted that if the magnitude and phase characteristics are, respectively, symmetric and antisymmetric around a centre frequency, then the complex SRC can be obtained by modulating a certain real SRC to the given frequency, which simplifies the implementation considerably. On the other hand, if an arbitrary frequency response, such as unequal stopband attenuations, is required, the complex SRC offers greater freedom and flexibility for processing the complex signals.

The brief is organized as follows. Section II is devoted to the design of CVDFs using either minimax or LS design criteria. Design examples, including the design of SRC for complex-valued signals, are given in Section III to demonstrate the effectiveness of the proposed approach. Finally, conclusion is drawn in Section IV.

\section{PROBlEM FoRMULATION}

\section{A. Complex-Valued Variable Digital Filter Design}

In a VDF [2], the desired response $H_{d}(\omega, \phi)$ is a function of a spectral parameter $\phi$ (also known as tuning or control parameters). The spectral characteristics of a VDF can therefore be continuously varied by changing the parameter $\phi$. The impulse response of the VDF is assumed to be a linear combination of a polynomial basis function of the spectral parameter $\phi$ and subfilter coefficients $h_{l}(n)$, and it is given by

$$
h(n, \phi)=\sum_{l=0}^{L-1} h_{l}(n) \cdot \phi^{l}
$$

where $\phi$ is assumed to vary linearly over a finite interval. From our experience, the closer the range of $\phi$ to zero (say $\phi \in[0,1]$ ), the smaller will be the dynamic range of the subfilter coefficients and it simplifies the multiplier-less realization of the VDFs [2]. The z-transform of the polynomial-based VDF is then given by

$$
H(z, \phi)=\sum_{l=0}^{L-1}\left[\sum_{n=0}^{N-1} h_{l}(n) z^{-n}\right] \cdot \phi^{l}=\sum_{l=0}^{L-1} H_{l}(z) \cdot \phi^{l}
$$

where $H_{l}(z)=\sum_{n=0}^{N-1} h_{l}(n) z^{-n}$ is the lth FIR subfilter. (2.2) suggests a very useful structure for implementing VDF called the Farrow's structure, which is shown in Fig. 1. Suppose that the impulse response of the $l$ th complex subfilter is given by $h_{l}(n)=h_{R, l}(n)+j h_{I, l}(n)$ for $n=0,1, \ldots, N-1$. By defining

$$
\begin{aligned}
& \boldsymbol{a}=\left[\boldsymbol{h}_{R, \mathbf{0}}^{T}, \ldots, \boldsymbol{h}_{R, L-1}^{T}, \boldsymbol{h}_{I, \mathbf{0}}^{T}, \ldots, \boldsymbol{h}_{I, L-1}^{T}\right]^{T} \\
& \boldsymbol{h}_{R, l}=\left[h_{R, l}(0), \ldots, h_{R, l}(N-1)\right]^{T} \\
& \boldsymbol{h}_{I, l}=\left[h_{I, l}(0), \ldots, h_{I, l}(N-1)\right]^{T}, \\
& \quad \text { for } l=0,1, \ldots, L-1 \\
& \boldsymbol{c}_{2}(\omega, \phi)=\left[\boldsymbol{c}_{1}(\omega, \phi)^{T}, \boldsymbol{s}_{1}(\omega, \phi)^{T}\right]^{T} \\
& \boldsymbol{s}_{2}(\omega, \phi)=\left[\boldsymbol{s}_{1}(\omega, \phi)^{T},-\boldsymbol{c}_{1}(\omega, \phi)^{T}\right]^{T} \\
& \boldsymbol{c}_{1}(\omega, \phi)=\left[\boldsymbol{c}(\omega)^{T}, \phi^{1} \cdot \boldsymbol{c}(\omega)^{T}, \ldots, \phi^{L-1} \cdot \boldsymbol{c}(\omega)^{T}\right]^{T} \\
& \boldsymbol{s}_{1}(\omega, \phi)=\left[\boldsymbol{s}(\omega)^{T}, \phi^{1} \cdot \boldsymbol{s}(\omega)^{T}, \ldots, \phi^{L-1} \cdot \boldsymbol{s}(\omega)^{T}\right]^{T} \\
& \boldsymbol{c}(\omega)=[1, \cos \omega \ldots, \cos ((N-1) \omega)]^{T} \\
& \boldsymbol{s}(\omega)=[0, \sin \omega \ldots, \sin ((N-1) \omega)]^{T} .
\end{aligned}
$$

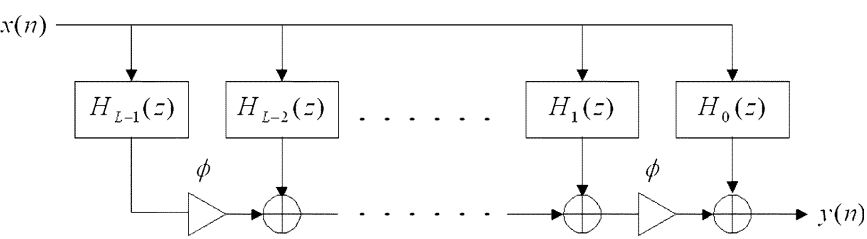

Fig. 1. General Structure of an FIR VDF.

Equation (2.2) can be written as

$$
H\left(e^{j \omega}, \phi\right)=\boldsymbol{a}^{T}\left\{\boldsymbol{c}_{2}(\omega, \phi)-j \boldsymbol{s}_{2}(\omega, \phi)\right\} .
$$

First of all, we approximate the desired response $H_{d}(\omega, \phi)$ by $H\left(e^{j \omega}, \phi\right)$ in the minimax sense by minimizing

$$
\begin{array}{r}
E_{\infty}=\max _{\Omega}\left\{W(\omega, \phi)\left|H\left(e^{j \omega}, \phi\right)-H_{d}(\omega, \phi)\right|\right\}, \\
(\omega, \phi) \in \Omega
\end{array}
$$

where $W(\omega, \phi)$ is a positive weighting function, and $\Omega$ is the (frequency, tuning range) of interest. Let $H_{R_{-} d}(\omega, \phi)$ and $H_{I_{-} d}(\omega, \phi)$ be the real and imaginary parts of $H_{d}(\omega, \phi)$, the minimization problem of $E_{\infty}$ in (2.5) can be reformulated as

$$
\min _{\boldsymbol{a}} \delta \text { subject to } \delta-\left[\alpha_{R}^{2}(\omega, \phi)+\alpha_{I}^{2}(\omega, \phi)\right]^{1 / 2} \geq 0
$$

for $(\omega, \phi) \in \Omega$, where $\alpha_{R}(\omega, \phi)=W(\omega, \phi)\left[\boldsymbol{a}^{T} \boldsymbol{c}_{2}(\omega, \phi)-\right.$ $\left.H_{R_{-} d}(\omega, \phi)\right]$ and $\alpha_{I}(\omega, \phi)=W(\omega, \phi)\left[\boldsymbol{a}^{T} \boldsymbol{s}_{2}(\omega, \phi)+\right.$ $\left.H_{I_{-} d}(\omega, \phi)\right]$. Digitizing the frequency variable $\omega$ and control parameter $\phi$ over a dense set of frequencies $\left\{\omega_{i}, 1 \leq i \leq K_{1}\right\}$ and control parameters $\left\{\phi_{k}, 1 \leq k \leq K_{2}\right\}$ in the range of interests, the constraints in (2.6) become $\delta-\left[\alpha_{R}^{2}\left(\omega_{i}, \phi_{k}\right)+\alpha_{I}^{2}\left(\omega_{i}, \phi_{k}\right)\right]^{1 / 2} \geq 0$. Moreover, by defining the augmented variable $\boldsymbol{x}=\left[\delta \boldsymbol{a}^{T}\right]^{T},(2.6)$ can be cast to the standard SOCP problem as follows:

$$
\min _{\boldsymbol{x}} \boldsymbol{c}^{T} \boldsymbol{x} \text { subject to } \boldsymbol{c}^{T} \boldsymbol{x} \geq\left\|\boldsymbol{F}_{\boldsymbol{i}, k} \boldsymbol{x}-\boldsymbol{g}_{i, k}\right\|_{2}
$$

where

$$
\begin{aligned}
\boldsymbol{c}= & {[1,0, \ldots 0]^{T}, \quad \boldsymbol{F}_{i, k}=W\left(\omega_{i}, \phi_{k}\right) } \\
& \cdot\left[\begin{array}{ll}
0 & \boldsymbol{c}_{2}\left(\omega_{i}, \phi_{k}\right)^{T} \\
0 & \boldsymbol{s}_{2}\left(\omega_{i}, \phi_{k}\right)^{T}
\end{array}\right] \\
\boldsymbol{g}_{i, k}= & W\left(\omega_{i}, \phi_{k}\right) \cdot\left[H_{R_{-} d}\left(\omega_{i}, \phi_{k}\right)-H_{I_{-} d}\left(\omega_{i}, \phi_{k}\right)\right]^{T} .
\end{aligned}
$$

Alternatively, (2.5) can be formulated as a SDP problem [8], [15], which might provide more flexibility but requires a longer design time. For simplicity, only the SOCP formulation is considered below. Instead of using the minimax criterion, the following LS design criterion can be minimized:

$$
E_{\mathrm{LS}}=\int_{\Omega} W(\omega, \phi) \cdot\left|H\left(e^{j \omega}, \phi\right)-H_{d}(\omega, \phi)\right|^{2} d \omega d \phi .
$$

$E_{\mathrm{LS}}$ can also be written as a quadratic function of $\boldsymbol{a}$ as

$$
\begin{aligned}
E_{\mathrm{LS}} & =\boldsymbol{a}^{T} \boldsymbol{Q} a-2 \boldsymbol{a}^{T} \boldsymbol{p}+k \\
& \left.=\left\|\boldsymbol{Q}^{1 / 2} \boldsymbol{a}-\boldsymbol{Q}^{-1 / 2} \boldsymbol{p}\right\|_{2}^{2}-\boldsymbol{p}^{T} \boldsymbol{Q}^{-1} \boldsymbol{p}+k\right)
\end{aligned}
$$

where

$$
\begin{aligned}
\boldsymbol{Q}= & \int_{\Omega} W(\omega, \phi) \cdot\left[\boldsymbol{c}_{2}(\omega, \phi) \boldsymbol{c}_{2}(\omega, \phi)^{T}\right. \\
& \left.+\boldsymbol{s}_{2}(\omega, \phi) \boldsymbol{s}_{2}(\omega, \phi)^{T}\right] d \omega d \phi \\
\boldsymbol{p}= & \int_{\Omega} W(\omega, \phi)\left[\boldsymbol{c}_{2}(\omega, \phi) H_{R_{-} d}(\omega, \phi)\right. \\
& \left.+\boldsymbol{s}_{2}(\omega, \phi) H_{I_{-} d}(\omega, \phi)\right] d \omega d \phi
\end{aligned}
$$


and $k=\int_{\Omega} W(\omega, \phi)\left|H_{d}(\omega, \phi)\right|^{2} d \omega d \phi$. The optimal LS solution is given by $a_{\mathrm{LS}}=Q^{-1} \boldsymbol{p}$. Alternatively, the minimization problem of $E_{\mathrm{LS}}$ can be formulated as the following SOCP:

$$
\min _{\boldsymbol{x}} \boldsymbol{c}^{T} \boldsymbol{x} \text { subject to } \boldsymbol{c}^{T} \boldsymbol{x}-\bar{k} \geq\|\overline{\boldsymbol{Q}} \boldsymbol{x}-\overline{\boldsymbol{p}}\|_{2}
$$

where $\overline{\boldsymbol{Q}}=\left[\boldsymbol{O}_{N} \boldsymbol{Q}^{1 / 2}\right], \overline{\boldsymbol{p}}=\boldsymbol{Q}^{-1 / 2} \boldsymbol{p}$, and $\boldsymbol{O}_{D}$ is a $D$ row zero vector. It can be seen that the number of constraints in the LS design is considerably less than the minimax design criterion, which usually leads to lower design complexity.

\section{B. Imposing Multiple Zero in the Stopband}

When designing digital filters, it is sometimes required to impose certain constraints on the frequency characteristics. An example is a prescribed number of zeros in the stopband, which are desirable in suppressing the alias components in sample rate converters. These constraints, which are linear equality constraints, can be obtained by equating the derivatives of the design response and its ideal counterparts as follows:

$$
\left.\frac{d^{v}}{d \omega^{v}} H\left(e^{j \omega}, \phi\right)\right|_{\omega=\hat{\omega}_{s}}=0, \quad v=0,1, \ldots, V_{\hat{\omega}_{s}}-1
$$

Substituting (2.2) into (2.11), we have

$\sum_{n=0}^{N-1} \sum_{l=0}^{L-1} n^{v} \cdot h_{l}(n) \cdot \phi^{l} e^{-j \hat{\omega}_{s} n}=0, \quad v=0,1, \ldots, V_{\hat{\omega}_{s}}-1$

To satisfy (2.12) over the entire range of the tuning parameter $\phi$, the following constraints are imposed to each subfilter:

$$
\sum_{n=0}^{N-1} n^{v} \cdot h_{l}(n) \cdot e^{-j \hat{\omega}_{s} n}=0, \quad v=0,1, \ldots, V_{\hat{\omega}_{s}}-1 .
$$

The constraints in (2.13) can be written as the following matrix representations for $\boldsymbol{h}_{R, l}$ and $\boldsymbol{h}_{I, l}$, respectively:

$$
\boldsymbol{A}_{\beta, l} \boldsymbol{h}_{\beta, l}=\boldsymbol{O}_{V_{\hat{\omega}_{s}}}, \quad l=0,1, \ldots, L-1
$$

where $\left[\boldsymbol{A}_{\beta, l}\right]_{v, n}=n^{v} e^{-j \hat{\omega}_{s} n}$ for $v=0,1, \ldots, V_{\hat{\omega}_{s}}-1$, and $\beta$ represents either $R$ or $I$. Here, $[\boldsymbol{A}]_{m, n}$ denotes the $(m, n)$ th entry of matrix $\boldsymbol{A}$. It should be noted that the above constraints can be imposed at more than one frequency point as long as the total number of linear equality constraints does not exceed the filter length $N$. Otherwise, there will be insufficient freedom in the filter coefficients to satisfy all these constraints. To incorporate them to the SOCP in (2.7) or (2.10), we combine all the constraints in (2.14) to form

$$
\boldsymbol{A} a=\boldsymbol{O}_{2 L V_{\hat{\omega}_{s}}}
$$

where $A=\operatorname{diag}\left\{A_{R, 0}, \ldots, A_{R, L-1}, A_{I, 0}, \ldots, A_{I, L-1}\right\}$. The minimization problems in (2.7) and (2.10) can be solved subject to these linear equality constraints using SOCP.

\section{Peak Error and Convex Quadratic Constraints}

To avoid excessive sidelobes of the LS solution, additional peak constraints can be imposed to the stopband. Let $\delta_{p}$ be the peak ripple to be imposed in a frequency band $\omega \in\left[\omega_{1}, \omega_{2}\right]$ (a collection of frequency bands is also feasible), then the peak error constraint can be written as

$$
\left|H\left(e^{j \omega}, \phi\right)\right| \leq \delta_{p}, \quad \omega \in\left[\omega_{1}, \omega_{2}\right], \quad(\omega, \phi) \in \Omega .
$$

Similar to the minimax formulation (2.16) can be rewritten as

$$
\delta_{p} \geq\|\boldsymbol{R} x\|_{2}, \text { where } \boldsymbol{R}=\left[\begin{array}{cc}
0 & \boldsymbol{c}_{2}(\omega, \phi)^{T} \\
0 & \boldsymbol{s}_{2}(\omega, \phi)^{T}
\end{array}\right] .
$$

After digitizing $\omega$ and $\phi$ over the range of interest, the resulting constraints can be augmented to the existing constraints in (2.7) and (2.10) for the minimax and LS criterion, respectively.

\section{Design of Complex SAmpling Rate Converter}

The design of programmable SRCs with arbitrary conversion factors was studied in detail by Ramstad [17]. In general, there are two approaches to implement a SRC with different tradeoff between the sampling rate and the hardware complexity. One is to up-sample the input signal by a factor of $L$, i.e., inserting $L-1$ zeros between successive time samples. This creates $L-1$ images in the frequency domain, which are then removed by an interpolation filter. If $L$ is sufficiently large, further interpolation with an irrational downsampling ratio can be achieved simply by a low-order interpolator, such as a cubic interpolator. The interpolator is controlled by a tuning parameter, which is able to provide rather accurate fractional delays up to a certain frequency, say $0.1 \pi$. After which, both the amplitude and phase responses deviate considerably from an ideal fractional-delay digital filter (FDDF) [4], [5]. Alternatively, these functions can also be implemented using a VDF [2], [11] with a control parameter $\phi$. For modest downsampling ratios, the VDF-based SRC is more efficient than the former approach because its coefficients can be jointly optimized to fulfill the given spectral and fractional-delay specifications [8]. For larger sampling conversion factors, a combination of these approaches can be used.

Similar arguments also hold for sample rate conversion of complex-valued signals, except that its frequency spectrum is not necessarily symmetric about $\omega=0$. To accommodate this asymmetric spectrum, we assume that the filter coefficients are complex quantities, which can be optimized using the proposed design method. The complex VDF-based SRC has the following ideal frequency response:

$$
H_{d}\left(e^{j \omega}, \phi\right)= \begin{cases}e^{-j \omega \tau(\phi)}, & \Omega_{p} \in\left[\omega_{p 1}, \omega_{p 2}\right] \\ 0, & \Omega_{s} \in\left[-\pi, \omega_{s 1}\right] \cup\left[\omega_{s 2}, \pi\right]\end{cases}
$$

where $\tau(\phi)=(N-1) / 2-D+\phi$ is the group delay of the VDF-based SRC; $D$ is the delay reduction parameter; $\Omega_{p}$ and $\Omega_{s}$ are the frequency of interest at the passband and stopband respectively; $\omega_{p 1}$ and $\omega_{p 2}\left(\omega_{s 1}\right.$ and $\left.\omega_{s 2}\right)$ are the passband (stopband) cutoff frequencies. Without loss of generality, we assume that $-\pi<\omega_{p 1}, \omega_{s 1}<0$, and $0<\omega_{p 2}, \omega_{s 2}<\pi$. In the passband of the CVDF, it behaves like a tunable fractional delay digital filter and the tuning parameter $\phi$ is used to provide the required arbitrary fractional delays. In the stopband, it helps to attenuate the undesirable frequency components. 

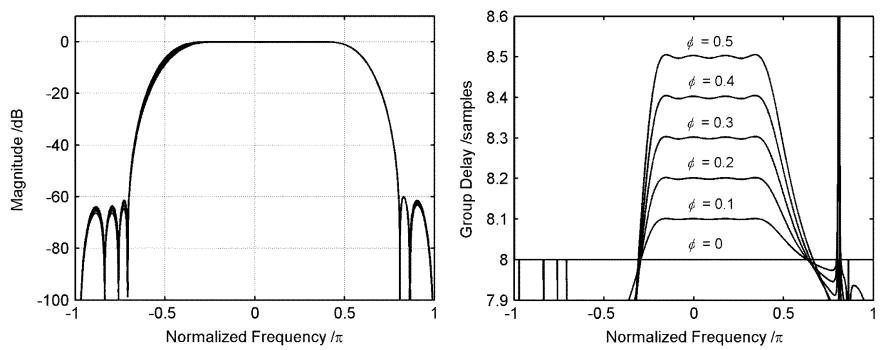

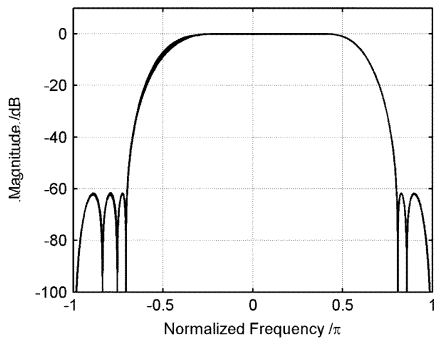

(c) (b)

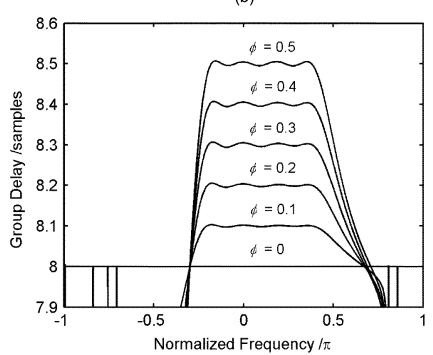

(d)

Fig. 2. (a) and (c) Frequency responses. (b) and (d) Group delays of the VDF-based SRC with $\phi=\{0,0.1,0.2,0.3,0.4,0.5\}$ using minimax and LS criteria, respectively.

Design Example 1: We now consider the design of CVDFbased SRCs using the proposed method. All the design problems were solved by the SeDuMi Matlab Toolbox [18] on a Pentium IV personal computer. The specifications are: 60-dB stopband attenuation, 0.025 samples group delay error in passband, $\omega_{p 1}=-0.2 \pi, \omega_{p 2}=0.4 \pi, \omega_{s 1}=-0.7 \pi, \omega_{s 2}=0.8 \pi$, and $\phi \in[-0.5,0.5]$. As an illustration, two zeros at $\omega=\pi$ are imposed in our CVDFs: i.e., $V_{\pi}=2$. It was found that a CVDF with $N=17, L=5, D=0$ (linear-phase) is able to meet the required specification and $K_{1}=100$ and $K_{2}=50$ grid points are found to give sufficiently accurate results. The frequency and group delay responses of the CVDF obtained by the proposed method and the minimax criterion are shown in Fig. 2(a) and (b), respectively. The corresponding passband deviation and stopband attenuation are respectively $0.0076 \mathrm{~dB}$ and $60.75 \mathrm{~dB}$. The computational time is about 15.98 minutes. Alternatively, we can solve the above problem using the LS criterion at a much lower design time of 0.03 minutes. To further reduce the sidelobe of the LS solution, peak stopband constraints are imposed with 40 samples for $\omega$ and 50 samples for $\phi$ near the transition band. Fig. 2(c) and (d) show the frequency and group delay responses of the CVDF so obtained. In exchange for a slightly lower performance at the unconstrained frequency bands, the maximum stopband attenuation is now increased to $60 \mathrm{~dB}$, compared to $54.65 \mathrm{~dB}$ for the conventional LS design. The time required is longer than the LS approach, but it is still much lower than that using the minimax approach. Table I summarizes the design results in this example. It should be noted that the proposed approach is able to design CVDFs with lower system delay by setting $D>0$ in (3.1). However, details are omitted due to page limitation.

As mentioned earlier, the SRC can be cascaded with a multistage decimator to form a programmable decimator [8]. Fig. 3(a) shows the general structure of the overall SRC. The basic idea is to insert the multistage decimator prior to the VDF-based $\mathrm{SRC}$, which is designed to support a down-sampling ratio of
TABLE I

DESIGN RESUlts OF COMPLEX VARIABLE DigITAL FILTERS

\begin{tabular}{c|c|c|c}
\hline \hline Design criteria & Minimax & LS & LS \\
\hline $\boldsymbol{V}_{\boldsymbol{\pi}}$ & 2 & 2 & 2 \\
\hline Peak stopband & N/A & N/A & $-60 \mathrm{~dB}$ \\
\hline Passband deviation (dB) & 0.0076 & 0.0100 & 0.0143 \\
\hline Stopband attenuation (dB) & 60.75 & 54.65 & 60 \\
\hline Group delay error (samples) & 0.0232 & 0.0235 & 0.0246 \\
\hline SOCP design time (min) & 15.98 & 0.03 & 4.76 \\
\hline \hline
\end{tabular}

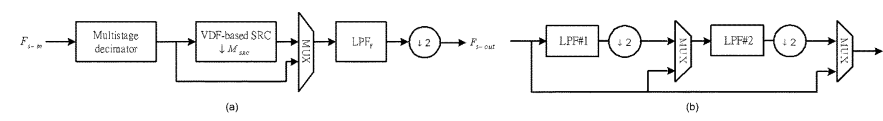

Fig. 3. (a) Overall structure of SRC. (b) Multistage decimator.

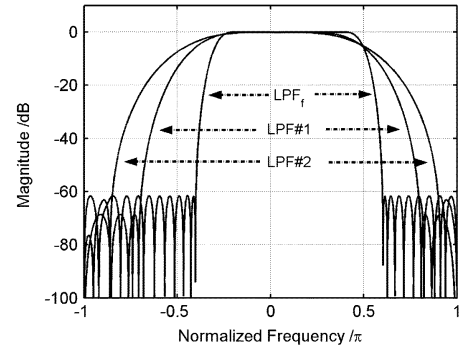

Fig. 4. Frequency responses of LPF\#1, LPF\#2, and $\mathrm{LPF}_{\mathrm{f}}$.

$M_{\mathrm{SRC}} \in(1,2)$, so as to provide larger decimation ratio to the input signals. For the two-stage decimator showed in Fig. 3(b), the decimation ratio $M^{*}$ of the overall SRC will lie between 2 and 16 . Higher decimator ratio can be achieved by using more decimation stages. In order to remove the residue interference from adjacent channels, the output of the SRC is fed to an additional anti-aliasing lowpass filter, denoted by $\mathrm{LPF}_{\mathrm{f}}$ in Fig. 3(a). It can be seen that the overall downsampling ratio $M^{*}$ is then given by $M^{*}=M_{\mathrm{SRC}} \cdot 2^{k}$, where $k$ is the number of 2-to-1 decimators selected.

Design Example 2: As an illustration, a two-stage decimator as shown in Fig. 3(b), is considered with $M^{*}$ lying between 2 and 16. The target passband deviation and stopband attenuation of the overall SRC are respectively 0.2 and $60 \mathrm{~dB}$. Also, we assume that the complex-valued input signal occupies the frequency band in the interval $[-0.3 \pi, 0.5 \pi]$. For designing complex filters, a number of methods were available in the literature (please refer to [19] and the references therein). For convenience, the proposed SOCP formulation is employed by setting $L=1, \phi=0$, and $K_{2}=1$. The specifications and performances of the anti-aliasing lowpass filters designed in the minimax sense are summarized in Table II. Their frequency responses are shown in Fig. 4. Together with the CVDF designed in previous example, the overall SRC gives a passband deviation of $0.194 \mathrm{~dB}$ and a stopband attenuation of $60.13 \mathrm{~dB}$. The frequency responses of the resulting complex-valued SRC with different operating ranges are shown in Fig. 5. As mentioned earlier, if the magnitude and phase characteristics are respectively symmetric and antisymmetric around a centre frequency, then the complex SRC can be obtained by modulating a certain real-valued SRC to the given frequency. If an arbitrary frequency response is required, a general complex-valued filter is required. As an illustration, Fig. 6 shows the frequency and 


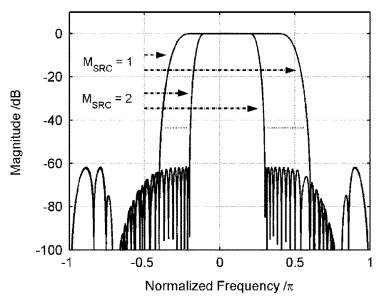

(a)

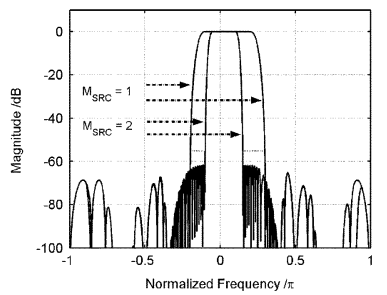

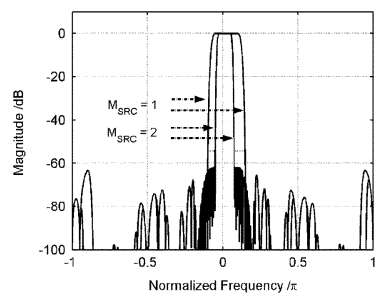

Fig. 5. Frequency responses of overall SRC with: (a) $2 \leq M^{*} \leq 4$, i.e., cascading $\operatorname{LPF}_{\mathrm{f}}$ and CVDF with $M_{\mathrm{SRC}} \in(1,2)$; (b) $4 \leq M^{*} \leq 8$, i.e., cascading $\mathrm{LPF} 2, \mathrm{LPF}_{\mathrm{f}}$ and the CVDF with $M_{\mathrm{SRC}} \in(1,2)$; and (c) $8 \leq \bar{M}^{*} \leq 16$, i.e., cascading LPF\#1, LPF\#2, $\mathrm{LPF}_{\mathrm{f}}$ and the CVDF with $M_{\mathrm{SRC}} \in(1,2)$.

TABLE II

SPECIFICATIONS AND Design Results of ANTi-Aliasing Low-PASS Filters.

\begin{tabular}{c|c|c|c}
\hline & LPF\#1 & LPF\#2 & LPF $_{\mathbf{f}}$ \\
\hline$\left(\omega_{p 1}, \omega_{p 2}\right)$ & $(-0.05 \pi, 0.1 \pi)$ & $(-0.1 \pi, 0.2 \pi)$ & $(-0.2 \pi, 0.4 \pi)$ \\
\hline$\left(\omega_{s 1}, \omega_{s 2}\right)$ & $(-0.85 \pi, 0.9 \pi)$ & $(-0.7 \pi, 0.8 \pi)$ & $(-0.4 \pi, 0.6 \pi)$ \\
\hline Filter length $N$ & 7 & 12 & 34 \\
\hline$V_{\pi}$ & 1 & 1 & 1 \\
\hline Passband deviation $(\mathrm{dB})$ & $6.02 \times 10^{-3}$ & $3.21 \times 10^{-3}$ & $7.26 \times 10^{-3}$ \\
\hline Stopband attenuation $(\mathrm{dB})$ & 63.21 & 68.04 & 61.66 \\
\hline \hline
\end{tabular}

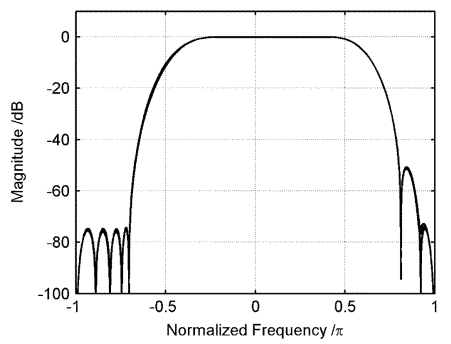

(a)

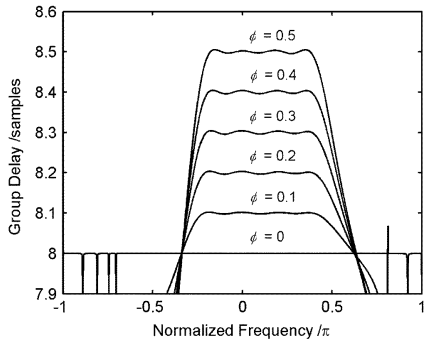

(b)
Fig. 6. (a) Frequency, and (b) group delay responses of the VDF-based SRC with unequal stopband attenuation.

group delay responses of a CVDF obtained by limiting the attenuation of the left-sided stopband to $75 \mathrm{~dB}$ and minimizing the remaining unconstrained bands using the proposed approach. One possible application is to provide different attenuation to different signal components in sensor array processing [20]. It can be seen that the CVDF has an asymmetry response about the center of its passband, which cannot be generated by modulating a real-valued VDF. To satisfy the same attenuation on the left-sided stopband, the length of a real-valued linear-phase VDF need to be increased from 17 to 20 , leading to a higher system delay.

\section{CONCLUSION}

A new method for designing LS and minimax optimal CVDFs with prescribed flatness and peak error constraints using SOCP is presented. Design examples are given to illustrate the effectiveness of the proposed approach. It shows that the proposed method offers more flexibility and good performance than conventional LS methods.

\section{REFERENCES}

[1] R. Zarour and M. M. Fahmy, "A design technique for variable digital filters," IEEE Trans. Circuits Syst. II, Analog Digit. Signal Process., vol. 36, no. 11, pp. 1473-1478, Nov. 1989.

[2] K. S. Pun, S. C. Chan, K. S. Yeung, and K. L. Ho, "On the design and implementation of FIR and IIR digital filters with variable frequency characteristics," IEEE Trans. Circuits Syst. II, Analog Digit. Signal Process., vol. 49, no. 11, pp. 689-703, Nov. 2002.

[3] P. Lowenborg and H. Johansson, "Linear programming design of linearphase FIR filters with variable bandwidth," in Proc. ISCAS'03, vol. 3, May 2003, pp. 554-557.

[4] C. W. Farrow, "A continuously variable digital delay element," in Proc. ISCAS'88, vol. 3, 1988, pp. 2641-2645.

[5] J. Vesma and T. Saramaki, "Design and properties of polynomial-based fractional delay filters," in Proc. ISCAS'00, vol. 1, May 2000, pp. 104-107.

[6] T. I. Laakso, V. Valimaki, M. Karjalainen, and U. K. Laine, "Splitting the unit delay, tools for fractional delay filter design," IEEE Signal Process. Mag., no. 1, pp. 30-60, Jan. 1996.

[7] H. Johansson and P. Lowenborg, "On the design of adjustable fractional delay FIR filters," IEEE Trans. Circuits Syst. II, Analog Digit. Signal Process., vol. 50, no. 4, pp. 164-169, Apr. 2003.

[8] S. C. Chan and K. S. Yeung, "On the application of variable digital filters (VDF) to the realization of software radio receivers," in Proc. ISCAS'03, vol. 3, May 2003, pp. 562-565.

[9] L. Erup, F. M. Gardner, and R. A. Harris, "Interpolation in digital modems. II. Implementation and performance," IEEE Trans. Commun., vol. 41, no. 6, pp. 998-1008, Jun. 1993.

[10] A. Tarczynski, G. D. Cain, E. Hermanowicz, and M. Rojewski, "WLS design of variable frequency response FIR filters," in Proc. ISCAS'97, vol. 4, 1997, pp. 2244-2247.

[11] W. S. Lu and T. B. Deng, "An improved weighted least-squares design for variable fractional delay FIR filters," IEEE Trans. Circuits Syst. II, Analog Digit. Signal Process., vol. 45, no. 9, pp. 689-685, Sep. 1997.

[12] M. S. Lobo, L. Vandenberghe, S. Boye, and H. Lebret, "Applications of second-order cone programming," Lin. Algeb. Appl., vol. 248, pp. 193-228, Nov. 1998.

[13] W. S. Lu and T. Hinamoto, "Optimal design of FIR frequency-response-masking filters using second-order cone programming," in Proc. ISCAS'03, vol. 3, May 2003, pp. 878-881.

[14] J. O. Coleman and D. P. Scholnik, "Design of nonlinear phase FIR filters with second-order cone programming," in Proc. MWSCAS'99, vol. 1, Aug. 1999, pp. 409-412.

[15] Handbook of semidefinite programming: Theory, Algorithms, and Applications, Kluwer, Norwell, MA, 2000.

[16] T. Hentschel and G. Fettweis, "Sample rate conversion for software radio," IEEE Commn. Mag., vol. 38, pp. 142-150, Aug. 2000.

[17] T. A. Ramstad, "Digital methods for conversion between arbitrary sampling frequencies," IEEE Trans. Acoust. Speech, Signal Process., vol. ASSP-32, pp. 577-591, 1984.

[18] J. F. Sturm, "Using SeDuMi 1.02, a MATLAB toolbox for optimization over symmetric cones," Optim. Math. Softw., vol. 11-12, pp. 625-653, 1999.

[19] L. J. Karam and J. H. McClellan, "Complex Chebyshev approximation for FIR filter design," IEEE Trans. Circuits Syst. II, Analog Digit. Signal Process., vol. 42, no. 3, pp. 207-216, Mar. 1995.

[20] A. G. Jaffer and W. E. Jones, "Constrained least-squares design and characterization of affine phase complex FIR filters," in Proc. 27th Asilomar Conf. Signals, Systems, Computers, vol. 1, Nov. 1993, pp. 685-691. 